\title{
APLASTIC ANAEMIA - THERAPEUTIC AND DEONTOLOGICAL ASPECTS
}

\author{
Andreea Ligia Dinca ${ }^{1}$, Oana Cristina Marginean ${ }^{1}$, Lorena Elena Melit ${ }^{1}$, \\ Raluca Damian², Mihaela Chincesan ${ }^{1}$ \\ ${ }^{1}$ Pediatrics Clinic 1, University of Medicine and Pharmacy of Targu-Mures \\ ${ }^{2}$ Neonatology Clinic 1, Emergency Clinical County Hospital of Targu-Mures
}

\begin{abstract}
Aplastic Anaemia is a severe potentially fatal haematological syndrome, characterised by peripheral pancytopenia and bone marrow hypoplasia, with an occurrence rate of 2-6 cases per million per year. This case report is about a 9 year-old boy, with no pathological known antecedents, and a sudden onset, apparently without any pathological associated conditions, who was diagnosed with severe Idiopathic Aplastic Anaemia, and a fulminate evolution. The lack of compliance of the parents regarding the therapy, and also the lack of a protective child's legislation, led to patient's death in a very short time.
\end{abstract}

Keywords: child, pancytopenia, aplastic anaemia

\section{INTRODUCTION}

Aplastic Anemia is described as a severe, potentially fatal, hematological syndrome, characterized by hypo or aplastic bone marrow associated to peripheral pancytopenia. It is a rare, sporadic, noncontagious condition, caused by the hematopoietic cell destruction inside the bone marrow and it may lead to a bone marrow failure (1).

Aplastic Anemia can be either inherited or acquired. The inherited type is quite rare and it is often associated to Fanconi Anemia or Congenital Dyskeratosis. Acquired form (over $80 \%$ of cases), can be caused by many possible triggers: autoimmune diseases, exposure to external/environmental factors; drug administration (Chloramphenicol!); viral infections (parvoviruses!), mycobacteria; severe deficiencies (B Vitamin, folate deficiency); Paroxysmal nocturnal hemoglobinuria, orthotopic liver transplantation - for fulminate hepatitis (3); pregnancy; or, $>50 \%$ of cases, idiopathic $(2,3,4)$. The most commonly accepted theory is the autoimmune hypothesis; aplastic anemia seems to develop secondary to the hematopoietic cell destruction by immune mechanisms $(5,6,7)$.
Symptoms result from the bone marrow failure effects: severe anemia, bleeding or infectious syndrome. There are signs suggesting inherited bone marrow failure syndrome such as: abnormal skin pigmentation, short stature, organ dysfunction, hypogonadism, skeletal anomalies $(8,9)$.

The positive diagnosis is based on the hematological exams. We must perform more than one complete blood counts revealing pancytopenia, in order to suggest the diagnosis; but the bone marrow examination will establish the certain diagnose. Severe aplastic anemia is a hematologic emergency, and care should be instituted as soon as possible. Clinicians must stress the need for patient's compliance with therapy for both the short and the long term prognosis. The first choice of treatment can be either pharmaceutical: immunosuppressive therapy, anti-thymocyte globulin, hematopoietic colony stimulating factor, antimetabolites, iron chelation therapy; or non-pharmaceutical: supportive care, blood transfusions or the bone marrow transplant. In extremely severe conditions, it may be necessary to proceed straight to emergent stem cell transplantation. In most cases this is the only curative treat- 
ment, but still it is conditioned by finding a matched sibling donor (ideally a brother or a sister. The estimated 10-year survival rate for the typical patient receiving immunosuppressive drugs is $68 \%$, compared with $73 \%$ for marrow transplant. However, there is a higher risk of relapse and late clonal disease for immunosuppressive drugs administration $(10,11)$.

\section{CASE PRESENTATION}

We present the case of a 9 year-old male patient, who was transferred from a territorial Emergency Unit in the Paediatrics Clinic 1 Targu-Mures, Compartment of Hemato-Oncology, complaining of altered general status, pallor of the skin and mucosa, severe drowsiness and spontaneous bleeding of the oral cavity. The mother related the onset of the pathology as being 4 days before the presentation, apparently consequent to a falling trauma. The family and personal history of the patient did not present anything relevant.

At the moment of admission, the general exam showed influenced general status, low weight: $23 \mathrm{~kg}-\mathrm{P}_{10}$; height: $130 \mathrm{~cm}-\mathrm{P}_{50}$, souring face, intense pallor, petechial lesions and ecchymosis on the trunk and limbs, asthenic thorax, tachycardia(VR: $130 \mathrm{bpm})$, spontaneous gums bleedings, gingival hematomas, liver under the right costal margin at 2 $\mathrm{cm}$, scrotal hematoma, without meningeal irritations signs. The complete blood count showed severe pancytopenia with Erythrocyte: $0.81 \mathrm{mil} / \mathrm{uL}$, Haemoglobin: 2.4 g/dL, Haematocrit: 6.7\%, Medium Erythrocytes Volume: $82.7 \mathrm{fL}$; White blood cells: 1300/uL, Neutrophils: 500/uL, Lymphocytes: 700/uL, PLT: < 1000/uL; Reticulocytes: 0.2\%; Peripheral smear showed hypocellularity; Erythrocyte Sedimentation Rate: $43 \mathrm{~mm} / \mathrm{h}$, Ferritin: 681.9 $\mathrm{ng} / \mathrm{mL}$, Alkaline phosphatase: $88 \mathrm{U} / \mathrm{L}$; the rest of the laboratory parameters being in normal range. Medullar exam: Mielocyte: 1\%, Metamielocyte: $1 \%$, Non-segmented: $2 \%$, Segmented: $6 \%$, Eosinophils: 2\%, Basophiles: 1\%, Small lymphocytes: 79\%, Monocytes: 1\%. Polychromatophil erythroblasts: 3\%, Oxiphil erythroblasts: 3\%, Plasmocytes: $1 \%$, Mastocytes: $1 / 100$; specimen with very low cellularity. Hypocellylarity of the MO (Fig. 1)

TORCH serology was negative, with the exception of IgG anti-CMV and IgG anti-HSV which were positive. The abdominal ultrasound showed mild hepatomegaly. The thoracic radiography did not identify any pathological elements. Bottom eye examination showed massive haemorrhages of the retina, near the papilla in both eyes. The cranial

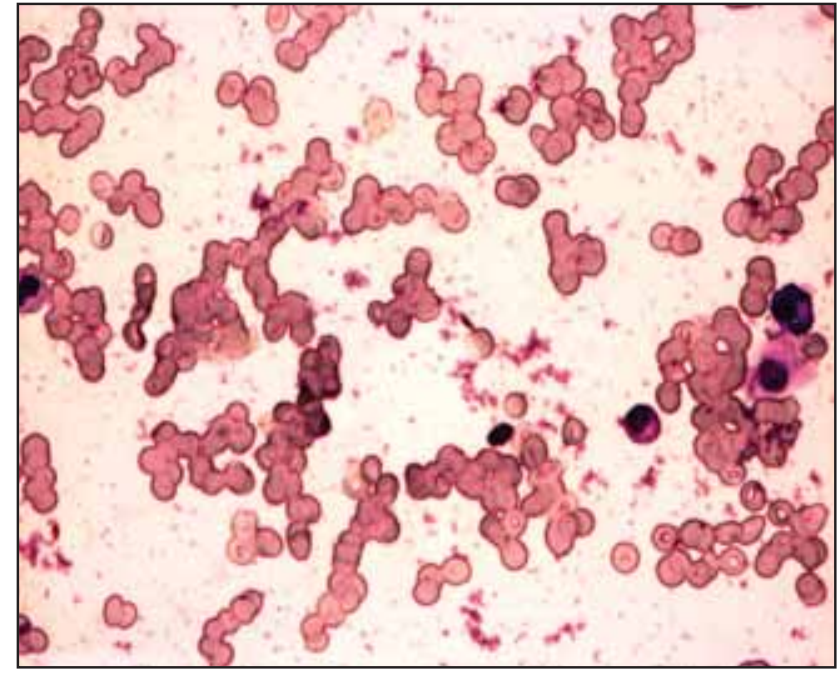

FIGURE 1. Low cellularity of the bone marrow

Computed Tomography exam was negative and the echocardiography revealed minim quantity of liquid in the pericardium.

Based on the clinical and the paraclinical exams, we established the diagnosis of Idiopathic Aplastic Anaemia, severe form. We initiated measurements of immediate intensive therapy, symptomatic treatment and prophylactic antibiotics with cephalosporin due to the severe neutropenia and the increased risk of opportunist infections. The parents were informed concerning the diagnosis, the options of treatment and the prognosis of the disease. We underlined the importance of performing the histocompatibility tests for possible allogenic medullar transplantation; thus, we discussed with a paediatric centre of transplantation in the country in order to transfer the patient. The mother refused to continue the investigations and the substitutive treatment despite the explications given by the physician, and she discharged the patient on her account 7 days after the admission. 8 days later, the patient accused oral bleedings, black stools and haematuria and he was readmitted with severe anaemia (Haemoglobin: 7.7 g/dL, Haematocrit: 27.6\%); severe thrombocytopenia - Platelets count: 10,000/ $\mathrm{uL}$ and Leucocytes: $1,300 / \mathrm{uL}$; the urine exam with microscopic haematuria. We administered blood derivate substances and hematopoietic colony stimulating factor until we managed to stabilize the patient; we explained the caregivers the treatment options and the investigations that were mandatory to be performed, but the mother's refuse hindered any intervention in this sense. We also contacted several times the Child's Protection Organisation. We initiated treatment with Medrol, Cyclosporine and auxiliary substances, with very slow therapeutic answer. In the next few weeks, the patient was 
readmitted several times in severe general status, but most times he was discharged at the mother's request, without being able to completely stabilise him. Last time he was discharged against medical advice, the laboratory investigations showed Haemoglobin: $8.3 \mathrm{~g} / \mathrm{dL}$, Haematocrit: 23.1\%; Reticulocytes: $0 \%$, Leucocytes: $1.17 / \mathrm{uL}$, Lymphocytes: 0.89/uL, Neutrophils: 0, Monocytes: 0.02/uL, Basophiles: 0.03/uL, Platelets: 50000/uL. Three days later, the patient returned in the Emergency Unit Tirgu-Mures in severe general status, with drowsiness, hemorrhagiparous syndrome and he died shortly with cardio-respiratory arrest, unresponsive to the resuscitation manoeuvres. The latter laboratory investigations from the day of death revealed liver and renal failure and severe pancytopenia.

\section{DISCUSSIONS}

Severe Aplastic Anemia is defined as a severe pancytopenia with at least two of the following criteria: absolute neutrophil count $<500 / \mathrm{mm}^{3}$, platelet count $<20.000 / \mathrm{mm}^{3}$, and reticulocyte count $<1 \%$ along with a hypocellularity of the bone marrow $<25 \%$ (1). The 2-year mortality rate for severe aplastic anaemia is approximately $80 \%$ with supportive care alone (12), the most common causes of death being severe bacterial sepsis or invasive fungal infections (13).

Our case is labeled as idiopathic, because the laboratory testing could not identify any etiological factor. Due to the parents' lack of compliance we could not perform vitamin $\mathrm{B}_{12}$ and folate dosage, a fetal hemoglobin test, a bone marrow biopsy, a genetic testing plus karyotyping, skeletal $\mathrm{x}$-Ray. Based on the absence of any abnormal skin or nail pigmentation, and also based on the laboratory investigations, we excluded an inherited form.

The first therapeutic choice is the stem cell transplantation, with a 5-year survival-rate of 70$80 \%$ (14). The supportive care and the immunosuppressive treatment, are an alternative in order to prevent the bleedings and the anemia symptoms, but we must properly evaluate the report between the risk to develop anti-Human Leucocyte antibodies or a hemochromatosis, and the short-term benefit $(15,16)$.
Considering this severe case and the fulminate evolution, our patient had a major indication for emergency marrow transplantation, especially that he had 2 possible compatible donors -2 healthy brothers; but the repeated parents' disallowance to test the 2 healthy brothers for histocompatibility, led us to immunosuppressive therapy that proved to be inefficient, therefore the patient died just a few weeks after the onset of the treatment. The parents' non-compliance made our therapeutic attempts extremely difficult and made us question the accuracy of administrating immunosuppressive at home. As regarding the repeated complaints to the Child's Protection, we would have necessitated a stronger assistance to parental counseling about the emergent need of marrow transplantation. We stress the need of a protective legislation that serves more to the needs of the children with potentially fatal diseases, and that constrains the parents to participate in an active way to any kind of treatment for the child's benefit, mostly when it can make the difference between life and death. The medical ethics and deontology are extremely important in the hemato-oncological pediatrics; these are the promoters of this battle against the system - whenever the benefit of the child does not come first, or, some other times, when there is a violation of a fundamental constitutional right of the child - the right to live, by the only person that should defend it by any means, the parents.

\section{CONCLUSIONS}

This case report describes a severe form of aplastic anemia, including the criteria above. The fulminate evolution and decease of a 9-years-old, apparently healthy, in less than 5 weeks from the onset, stresses the particularities of this case. The poor prognosis was due to the lack of compliance of the parents because in spite of the supportive care and the immunosuppressive therapy, the general status altered progressively. The parental compliance with the therapy, and also a more active and prompt involvement of the Child's Protection Organisation regarding these cases and also the introduction of a protective legislation on this matter are extremely important. 


\section{REFERENCES}

1. Philip Lanzkowsky; Manual of Pediatric Hematology and Oncology; Fifth Edition; Elsevier, 2011:123-167

2. Breakey V.R., Meyn S., Allen C., Dokal I., et al. Hepatitis-associated aplastic anemia presenting as a familial bone marrow failure syndrome. J Pediatr Hematol Oncol. 2009 Nov. 31(11):884-887.

3. Gonzalez-Casas R., Garcia-Buey L., Jones E.A., et al. Systematic review: hepatitis-associated aplastic anaemia - a syndrome associated with abnormal immunological function. Aliment Pharmacol Ther. 2009 Sep 1. 30(5):436-443.

4. Phillip Scheinberg, Michael Marte, Olga Nunez, et al. Paroxysmal nocturnal hemoglobinuria clones in severe aplastic anemia patients treated with horse anti-thymocyte globulin plus cyclosporine. Haematologica, Jul 2010, 95 (7): 1075-1080; DOI: 10.3324/ haematol.2009.017889

5. Solomou E.E., Keyvanfar K., Young N.S. T-bet, a Th1 transcription factor, is up-regulated in T cells from patients with aplastic anemia. Blood. 2006 May 15. 107(10):3983-3991.

6. Socié G., Rosenfeld S., Frickhofen N., et al. Late clonal diseases of treated aplastic anemia. Semin Hematol. 2000 Jan. 37(1):91-101.

7. Nakao S. Immune mechanism of aplastic anemia. Int J Hematol. 1997 Aug. 66(2):127-134.

8. Frickhofen N., Kaltwasser J.P., Schrezenmeier H., et al. Treatment of aplastic anemia with antilymphocyte globulin and methylprednisolone with or without cyclosporine. The German Aplastic Anemia Study Group. N Engl J Med. 1991 May 9. 324(19):1297-1304.
9. Doherty L., Sheen M.R., Vlachos A., et al. Ribosomal protein genes RPS10 and RPS26 are commonly mutated in Diamond-Blackfan anemia. Am J Hum Genet. 2010 Feb 12. 86(2):222-228.

10. Miano M., Dufour C. The diagnosis and treatment of aplastic anemia: a review. Int J Hematol. 2015 Jun. 101 (6):527-535.

11. Locasciulli A., Oneto R., Bacigalupo A., et al. Outcome of patients with acquired aplastic anemia given first line bone marrow transplantation or immunosuppressive treatment in the last decade: a report from the European Group for Blood and Marrow Transplantation (EBMT). Haematologica. 2007 Jan. 92(1):11-8.

12. Amy E. DeZern. Robert A. Brodsky. Clinical management of aplastic anemia. Expert Rev Hematol. 201;.4(2): 221-230

13. Judith C.W. Marsh, Sarah E. Ball, Jamie Cavenagh, et al. Guidelines for the diagnosis and management of aplastic anaemia. British Journal of Haematology. 2009; 147: 43-70

14. Young N.S. Current concepts in the pathophysiology and treatment of aplastic anemia. Hematology Am Soc Hematol Educ Program. 2013; 2013:76-81

15. Korthof E.T., Bekassy A.N., Hussein A.A. Management of acquired aplastic anemia in children. Bone Marrow Transplantation. 2013; 48:191-195

16. Seiji Kojima, Keizo Horibe, Jun Inaba, et al. Long-term outcome of acquired aplastic anaemia in children: comparison between immunosuppressive therapy and bone marrow transplantation. British Journal of Haematology. 2000; 111(1): 321-328 